\title{
Expression of vasorin (Vasn) during embryonic development of the mouse
}

\author{
A. Michaela Krautzberger ${ }^{\mathrm{a}, 1}$, Barbara Kosiol ${ }^{\mathrm{a}}$, Manuela Scholze ${ }^{\mathrm{a}, \mathrm{b}}$, Heinrich Schrewe ${ }^{\mathrm{a}, \mathrm{b}, *}$ \\ a Department of Developmental Genetics, Max Planck Institute for Molecular Genetics, Ihnestrasse 63-73, 14195 Berlin, Germany \\ ${ }^{\mathrm{b}}$ Institute for Medical Genetics, Charité-University Medicine Berlin, Campus Benjamin Franklin, Hindenburgdamm 30, 12203 Berlin, Germany
}

\section{A R T I C L E I N F O}

Article history:

Received 19 January 2012

Accepted 28 February 2012

Available online 8 March 2012

\section{Keywords: \\ Vasorin \\ Vasn \\ Slitl2 \\ Atia}

Transmembrane protein

Mouse embryo

Development

Expression pattern

Whole mount in situ hybridization

lacZ knock-in

Vascular smooth muscle cells

Skeletal system

Kidney

Lung

\begin{abstract}
A B S T R A C T
The murine vasorin (Vasn) gene, initially known as Slit-like 2, encodes a transmembrane protein that shares structural similarities with the eponymous Slit proteins. However, whether it also shares functional similarities with these large secreted proteins remains to be elucidated. Here, we report expression of Vasn during embryonic and fetal development of the mouse using whole-mount in situ hybridization (WISH) and histochemical detection of $\beta$-galactosidase expressed from a targeted Vasn $^{\text {lacz }}$ knock-in allele. Comparison of whole-mount staining patterns of both approaches showed identical expression domains, confirming that Vasn promoter-driven $\beta$-galactosidase expression faithfully reflects endogenous Vasn expression. Vasn is highly expressed in vascular smooth muscle cells (hence the name), a finding consistent with a previous report on its human homolog VASN, whose extracellular domain was shown to function as a TGF- $\beta$ trap (Ikeda et al., 2004). Most striking, however, is Vasn's prominent expression in the developing skeletal system, starting as early as the first mesenchymal condensations appear. Moreover, distinct expression domains outside the bones, e.g., in the developing kidneys and lungs, suggest further roles for this gene in the mouse. Recently, it was shown that mitochondria-localized Vasn protects cells from TNF $\alpha$ - and hypoxia-induced apoptosis, and partial deletion of the Vasn coding sequence leads to increased sensitivity of hepatocytes to TNF $\alpha$-induced apoptosis (Choksi et al., 2011). By providing a first comprehensive analysis of the Vasn expression pattern during mouse embryonic development, our study will help to further elucidate its biological functions.
\end{abstract}

(c) 2012 Elsevier B.V. All rights reserved.

\section{Results and discussion}

Vasorin (Vasn) was first isolated from a mouse kidney cDNA library. The locus consists of two exons spanning over a region of approximately $11 \mathrm{~kb}$ on mouse chromosome 16 . Its coding region is entirely located on exon 2, which translates into a typical single-pass type I transmembrane protein with a calculated molecular weight of $72 \mathrm{kDa}$. Based on the combination of leucine-rich repeats (LRRs) and an epidermal growth factor (EGF)-like motif - the defining feature of the Slit family of proteins - the gene was originally named Slit-like 2 (Slitl2). It is as yet unknown whether this protein shares overlapping functions with the eponymous Slit proteins, which are best known for their role in axon guidance (Ypsilanti et al., 2010). Vasn homologs have been identified in numerous other species. In zebrafish, vasn is expressed in embryonic midline structures of the central nervous system as well as in the vascular system (Chen et al., 2005). The human homolog VASN shares $84 \%$ sequence identity with the murine protein. It has been demon-

\footnotetext{
* Corresponding author. Tel.: +49 308413 1302; fax: +49 3084131216 .

E-mail address: schrewe@molgen.mpg.de (H. Schrewe).

1 Present address: Department of Stem Cell and Regenerative Biology, Harvard University, 7 Divinity Avenue, Cambridge, MA 02138, USA.
}

strated that VASN's extracellular domain negatively modulates TGF- $\beta$ signaling by sequestering the ligand in a complex, thereby preventing ligand-receptor interactions and subsequent downstream signaling (Ikeda et al., 2004; Malapeira et al., 2010). Proteomic analyses revealed VASN as potential biomarker in kidney disease and cancer (Caccia et al., 2011; Moon et al., 2011). In mice, Vasn expression increases gradually during embryonic development, as evidenced by Northern blot analysis, and in situ hybridization showed expression in developing and adult aortic vascular smooth muscle cells (Ikeda et al., 2004). Recently, it was demonstrated that Vasn protects cells against TNF $\alpha$ - and hypoxia-induced apoptosis through regulation of the mitochondrial antioxidant thioredoxin-2 (TRX2), and that mice with a partial deletion of the Vasn coding sequence show increased susceptibility to apoptosis in a model of TNF $\alpha$-induced hepatitis (Choksi et al., 2011).

To delineate in detail the tissues that may require Vasn during mouse development, we assayed endogenous Vasn mRNA expression using whole-mount in situ hybridization (WISH) from embryonic day 8.5 (E8.5) to E11.5. In addition, we generated a Vasn lacz knock-in reporter mouse strain. In these mice, the Vasn coding sequence is replaced by the $\beta$-galactosidase (lac $Z$ ) open reading frame fused to a nuclear localization signal on one allele, thereby placing lacZ under transcriptional control of the endogenous Vasn regula- 
tory elements (Fig. 1A). Correct integration of the targeting fragment in embryonic stem cells was confirmed by PCR (Fig. 1B). Cre-mediated recombination led to excision of the selection cassette, and heterozygous Vasn ${ }^{\text {lacz/+ }}$ mice were identified by PCR (Fig. 1C). Histochemical detection of $\beta$-galactosidase protein expression from the targeted allele in Vasn $n^{\text {lacz/+ }}$ embryos produced an expression profile consistent with the WISH analysis of endogenous Vasn expression from E8.5 to E11.5 and thus was further employed for detailed analysis of Vasn expression domains throughout embryonic development.

\subsection{Whole-mount expression analysis of Vasn}

\subsubsection{Comparison of WISH and X-gal staining patterns from E8.5 to} E11.5

In the E8.5 embryo, endogenous Vasn mRNA and Vasn promoter-driven reporter protein expression is detectable in the hindbrain and the midline of the neural tube. In addition, the lateral mesoderm and the caudal extremity exhibit staining (Fig. 2A and E). At E9.5, expression is prominent in the rhombic lip, a restricted region adjacent to the hindbrain roof plate. Cross sections show expression in the roof plate and, in addition, reveal staining in the floor plate. Moreover, the first branchial arches, the septum transversum, the emerging forelimb buds, and the lining of the coelomic cavity reveal staining (Fig. 2B-B" and F-F"). Wholemount specimens at E10.5 again show identical expression domains with both staining methods. Strong signal is seen throughout the head and trunk mesenchyme as well as in the branchial arches. Like the forelimb buds, the newly formed hindlimb buds are intensely stained (Fig. $2 \mathrm{C}$ and $\mathrm{G}$ ). The overall staining patterns essentially correspond to that of the previous stage at E11.5. However, upon further differentiation of the skeleton, clear Vasn transcript expression as well as $\beta$-galactosidase reporter activity is detectable along the vertebral axis (Fig. 2D and $\mathrm{H}$ ).
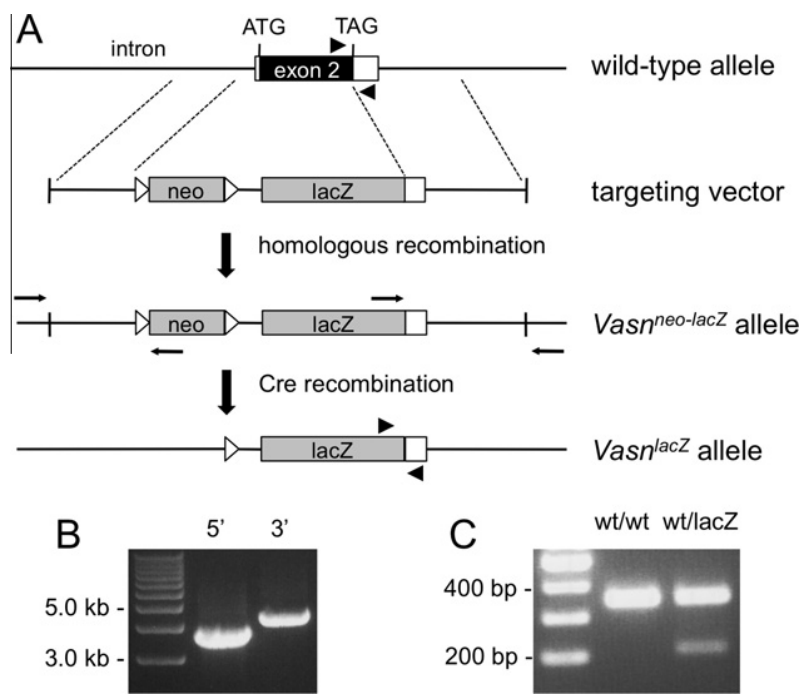

Fig. 1. Targeting of the Vasn gene to produce a $\operatorname{Vasn}^{\text {lacZ }}$ reporter strain (A) Targeting scheme showing wild-type allele and targeting construct used to produce the $V_{a s n^{\text {neo-lacz }}}$ allele by homologous recombination. The Vasn ${ }^{\text {lacz }}$ allele was generated by subsequent Cre-mediated excision of the selection cassette. (B) Results of longrange PCR confirming correct recombination of the targeting construct in ES cells. External forward/reverse neo primer and lacZ forward/external reverse primers used for 5' and 3' end, respectively, are indicated as arrows in (A). (C) Results of three-primer PCR to identify heterozygous Vasn lacz/+ embryos. Primers used for discrimination between the wild-type and $\operatorname{Vasn}^{\text {lacZ }}$ allele are indicated as arrowheads in $(A)$.

\subsubsection{X-gal staining patterns from E12.5 to E15.5}

Despite the reduced substrate penetration efficiency owing to an increasing skin thickness, X-gal-stained Vasn ${ }^{\text {lacZ/+ }}$ embryos from stages E12.5 to E15.5 all display striking reporter gene activity throughout the developing skeletal system. Here, signal is detected in the axial as well as the appendicular skeleton, e.g., in the vertebral bodies and the long bones of the limbs, respectively (Fig. 2I-L). As shown for E9.5, wild-type littermates at all stages examined do not exhibit any X-gal staining (Fig. 2M).

\subsection{Histological expression analysis of Vasn}

To identify Vasn expression domains on a cellular level, we have analyzed sagittal sections at early and middle stages of both organogenesis (E10.5 and E12.0, respectively) and fetal mouse development (E14.5 and E17.5, respectively) for $\beta$-galactosidase activity in heterozygous Vasn ${ }^{\text {lacz/+ }}$ embryos.

\subsubsection{E10.5}

Section analysis confirms lacz activity throughout the cephalic, branchial arch, and trunk mesenchyme (Fig. 3A). In the developing heart, reporter signal is mainly found in the myoepicardial layer of the outflow tract, while lacZ activity is barely detected in the cells of the common atrial and bulbus cordis region (Fig. 3B). Inferior to the common atrial chamber, the lung buds become evident at this stage of embryogenesis; $\beta$-galactosidase activity is not detectable in the epithelium of the developing main bronchi (Fig. 3C). Epithelial cells budding off from the floor of the primitive pharynx to form the thyroid primordium are devoid of X-gal staining (Fig. 3D). In the trunk region, strong signal is detected in the septum transversum, whereas the invading hepatoblasts appear unstained. In the adjacent cystic primordium, staining is most dense in the stalk cells (Fig. 3E). Reporter protein expression is notable in epithelial cells lining the mid- and hindgut (Fig. 3F). Similarly, Vasn promoter activity is evidenced by X-gal staining in mesonephric tubular cells (Fig. 3G).

\subsubsection{E12.0}

By E12.0, substantial changes have occurred with regard to the development of the future spine. While lacZ activity is barely detected in the newly formed dorsal root ganglia, the intervening, caudal condensed portions of the sclerotomes are clearly marked by $\beta$-galactosidase protein expression (Fig. $4 \mathrm{~A}$ and $\mathrm{B}$ ). Intense signal is also observed in the parietal and visceral mesothelial cells lining the coelomic cavities and internal organs, respectively. In the developing heart, for example, X-gal staining is most intense in the pericardium, but rather sparse in the ventricular trabeculated muscle and atrio-ventricular cushion tissue (Fig. 4A and C). Likewise, staining mostly localizes to the cells surrounding the hepatic parenchymal tissue, in which only scattered nuclear lacz activity is observed (Fig. 4C). The cells of the pancreatic primordia, on the other hand, display homogenous $\beta$-galactosidase activity (Fig. 4D). The urogenital system demonstrates a differential staining profile with sparse signal in the gonadal primordium at its medial aspect, but evenly distributed signal throughout the mesonephric tissue with its degenerating tubules at its lateral aspect (Fig. 4E). Homogenous nuclear $\beta$-galactosidase activity is also observed throughout the condensed metanephric mesenchyme and the ureteric bud stalk located at the caudal part of the urogenital ridge (Fig. 4F). Moreover, the wall of the dorsal aorta shows intense staining (Fig. 4G).

\subsubsection{E14.5}

As stated for the whole-mount analysis, X-gal staining is particularly prominent throughout all parts of the developing skeletal system from this stage onwards, including areas of both intramem- 


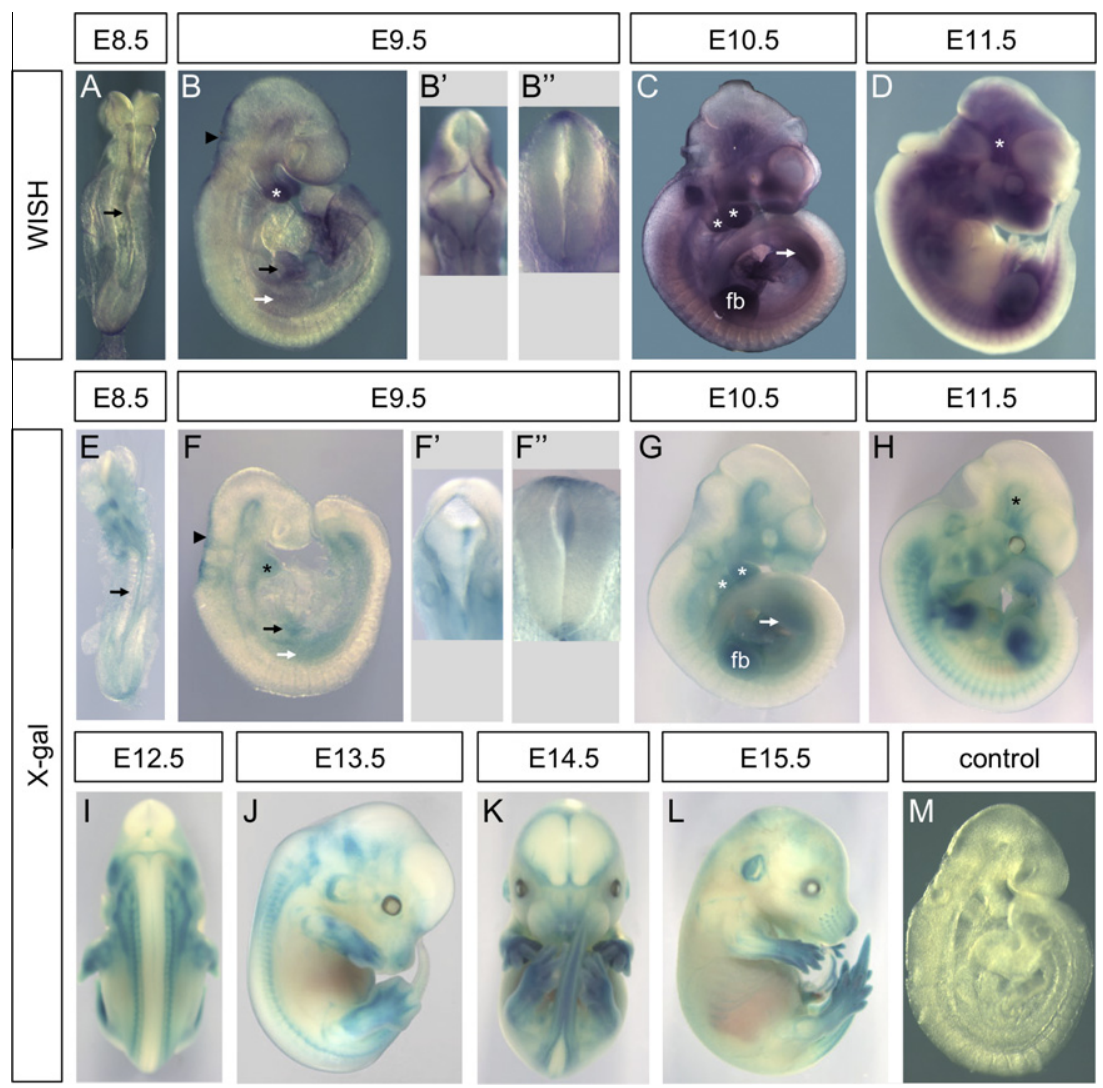

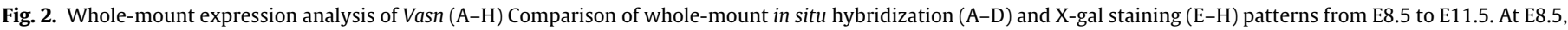

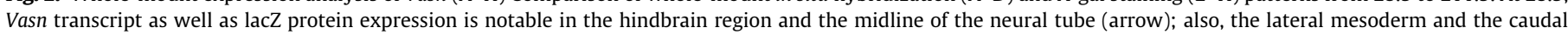

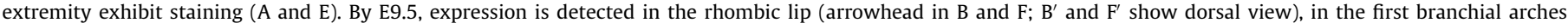

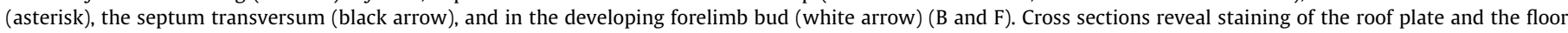

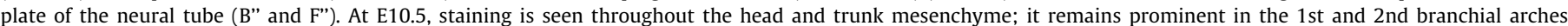

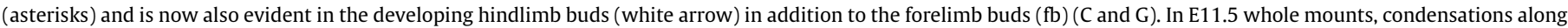

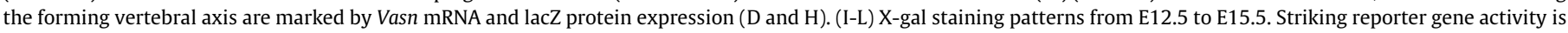
detected in both the developing axial and appendicular skeletal system. (M) Wild-type littermates (E9.5 shown) do not exhibit X-gal staining.

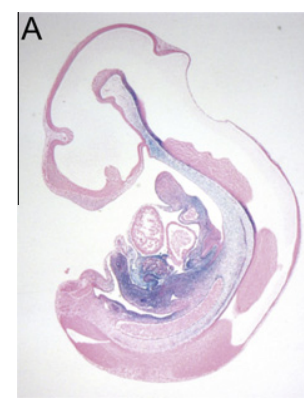

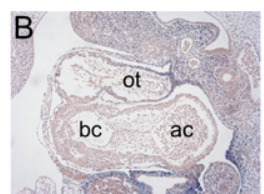

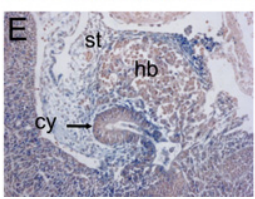

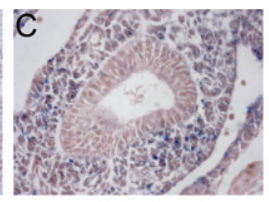
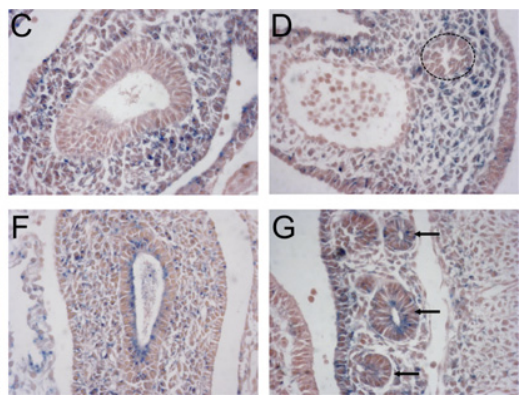

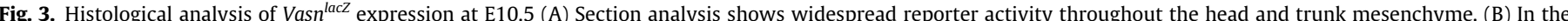

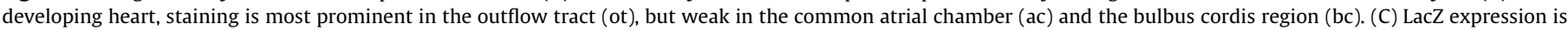

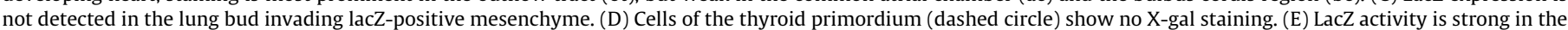

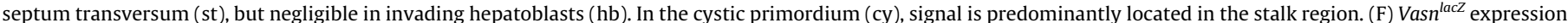

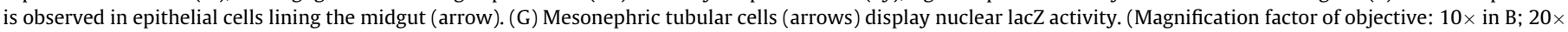
in $\mathrm{E} ; 40 \times$ in $\mathrm{C}-\mathrm{G})$.

branous and endochondral ossification (Fig. 5A). As opposed to the previous stage, dispersed lacZ activity is now also detectable in the dorsal root ganglia and - to a lesser extent - also within the cells of the developing brain and spinal cord, while being virtually absent in the pituitary gland primordium (Fig. $5 \mathrm{~B}$ and $\mathrm{C}$ ). In the two lobes of the thymus gland, quite substantial structures at this stage, lacZ activity is mostly localized in the capsule and the septa emerging from it (Fig. 5D). By E14.5, the lungs contain numerous primary, sec- ondary, and tertiary bronchi. While $\beta$-galactosidase activity is sparse in bronchial epithelial cells, X-gal staining persists in the pulmonary mesenchymal tissue and is most dense within vessel walls (Fig. 5E). The increasing degree of differentiation with the advancement of development can also be appreciated with regard to the regionalized staining in the kidney. In the primitive nephrons, marked Vasn promoter activity is observed in the proximal parts of the S-shaped bodies. In the adjacent adrenal glands, reporter pro- 


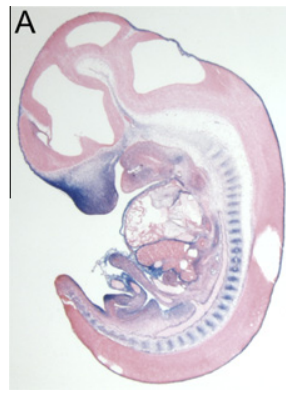

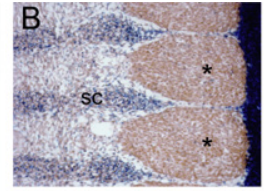

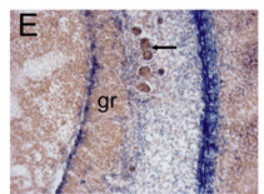

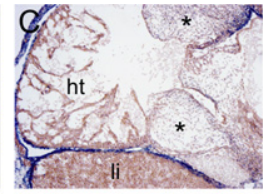

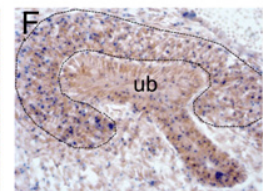

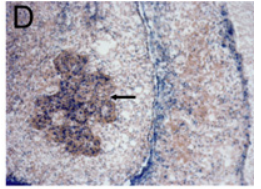

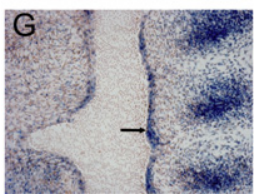

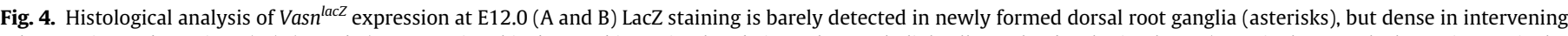

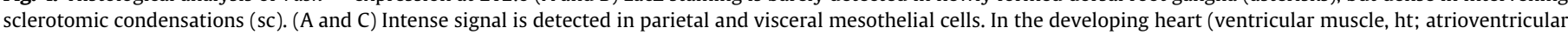

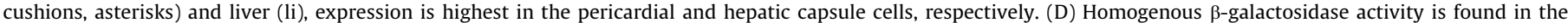

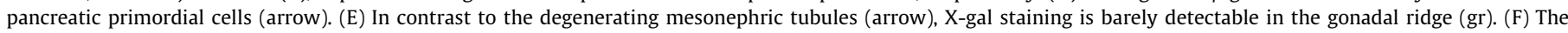

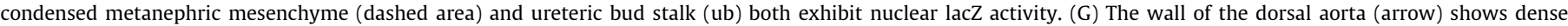
staining. (Magnification factor of objective: $10 \times$ in C; $20 \times$ in B, D, E and G; $40 \times$ in F).
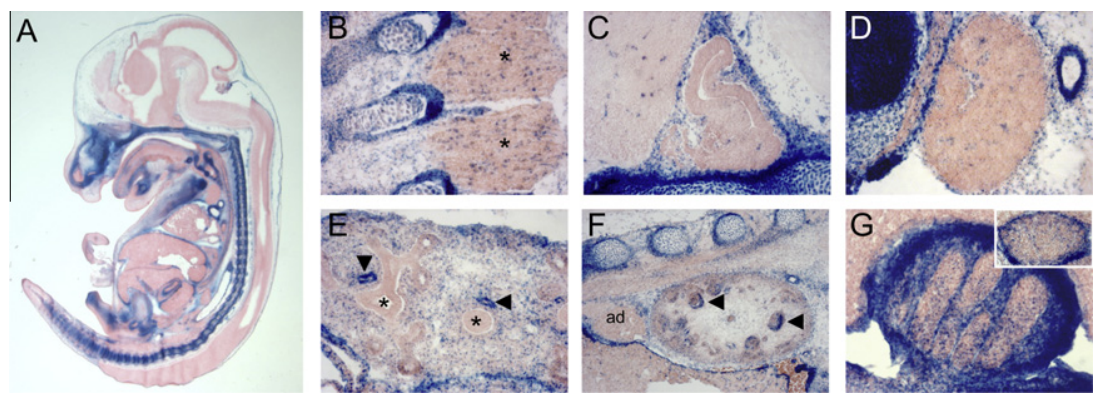

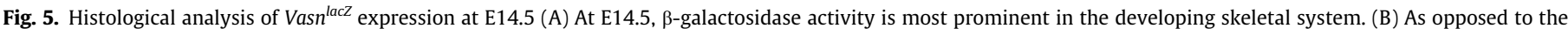

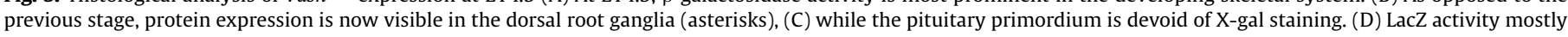

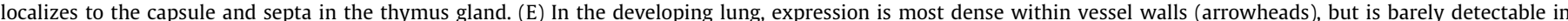

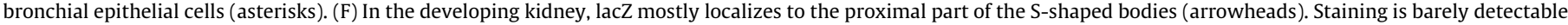

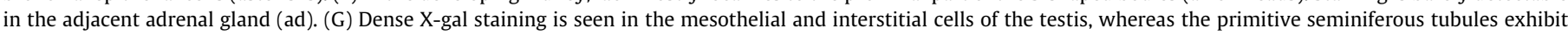

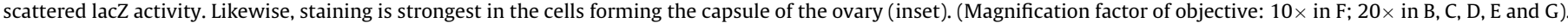

tein expression is barely detected in both the mesoderm-derived cortex and the ectoderm-derived medulla (Fig. 5F). Histological examination of a male embryo reveals scattered $\beta$-galactosidase activity in the cells of the primitive seminiferous tubules, while intense staining is found in the interstitial tissue as well as in the surrounding mesothelium. Similarly, X-gal staining is found to be dense within the cells forming the capsule of the ovary (Fig. 5G).

\subsubsection{E17.5}

$\mathrm{X}$-gal staining remains most prominent in the skeletal system also at E17.5 (Fig. 6A). By this stage of development, extensive areas of ossification have developed, e.g., within the shaft region of the long bones, where intense staining can be observed (Fig. 6B). Dramatic changes are also observed with regard to the appearance of the lungs compared to the previous stage, notably the formation of numerous alveolar ducts. Generalized low-level signal is detected throughout the airway epithelium lining the respiratory duct system; moreover, arterial vessel walls show strong staining in comparison to adjacent venous vessels (Fig. 6C). Regionalized $\beta$-galactosidase activity is apparent in the stomach with dense staining in the mucosa lining the gastric fundus, but low signal in its body region (Fig. 6D). Distinct staining is
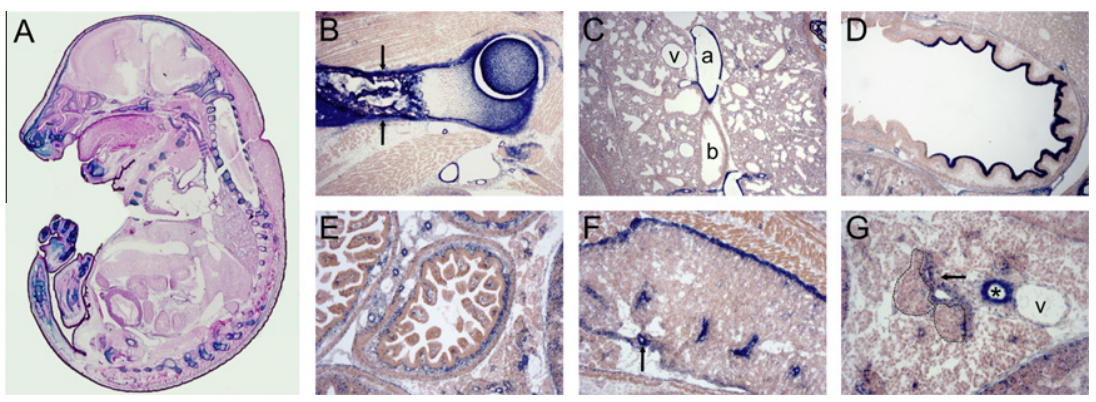

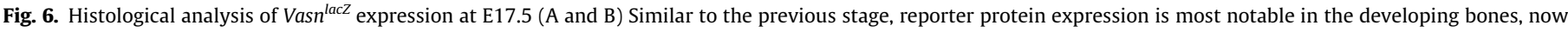

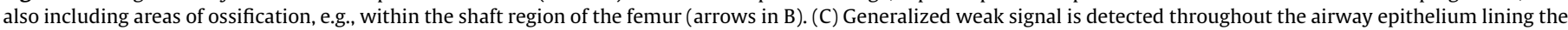

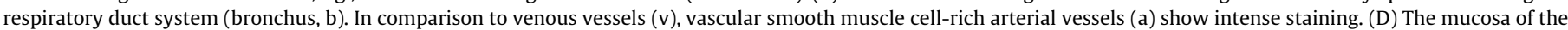

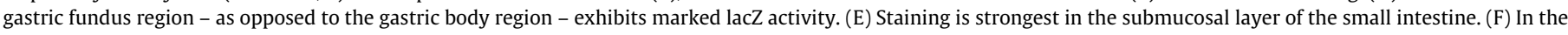

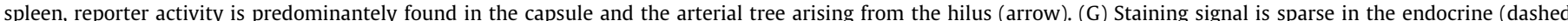

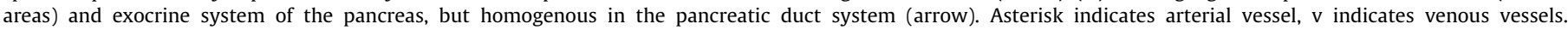
(Magnification factor of objective: $5 \times$ in $\mathrm{B}-\mathrm{D} ; 10 \times$ in $\mathrm{E}$ and $\mathrm{F} ; 20 \times$ in $\mathrm{G}$ ). 
also observed in the lower intestinal tract. Cross-sectioning of the small intestine reveals that lacZ mostly localizes to the submucosal layer (Fig. 6E). In the spleen, strong signal is evident in the capsule as well as in the arterial tree arising from the hilus, whereas the parenchymal tissue itself only exhibits sparse staining (Fig. 6F). Similarly, the arterial vessels in the pancreas exhibit strong $\beta$ galactosidase activity. Homogenous nuclear lacZ activity is seen in the pancreatic duct system, while staining is sparse in both the endocrine and exocrine components, i.e., in the islets of Langerhans and the acinar cells, respectively (Fig. 6G).

In this report, we have first characterized in detail the expression pattern of Vasn during mouse embryonic development. With various distinct expression domains, our results indicate important roles for Vasn e.g., in the developing skeletal system, the kidneys, and the lungs, and should thus help to further elucidate its biological functions.

\section{Experimental procedures}

\subsection{Whole-mount in situ hybridization}

For the generation of the probe, the plasmid pBluescript II KSVasn containing a $1.8 \mathrm{~kb}$-long fragment of the murine Vasn cDNA sequence (accession number AJ458938) was linearized with EcoRI, and anti-sense, digoxygenin (DIG)-labeled probe was obtained with the DIG RNA Labeling Kit (Roche) using T7 polymerase. In order to reduce probe length, it was incubated in hydrolysis buffer ( $40 \mathrm{mM} \mathrm{NaHCO}, 75 \mathrm{mM} \mathrm{Na}_{2} \mathrm{CO}_{3}, \mathrm{pH} 10.2$ ) for $4 \mathrm{~min}$ at $60^{\circ} \mathrm{C}$. Whole-mount in situ hybridization was performed according to the protocol of the "molecular anatomy of the mouse embryo project" (mamep) which is available online (http://mamep.molgen.mpg.de/protocol.html).

\subsection{Generation of Vasn ${ }^{\text {lacZ }}$ mice}

The Vasn ${ }^{\text {lacz }}$ knock-in allele was generated by targeted homologous recombination in G4 embryonic stem cells (George et al., 2007), thereby replacing the Vasn coding sequence on exon 2 with the $\beta$-galactosidase (lacZ) ORF fused to a nuclear localization signal. A loxP-flanked PGKneo cassette was inserted upstream to select for targeted clones. Correct integration was assessed using the Expand Long Template PCR System (Roche) according to the protocol of the European Conditional Mouse Mutagenesis Program (EUCOMM; http://www.eucomm.org) with primer pairs spanning the Vasn locus-targeting vector junctions on both sides. For the 5' junction, a forward primer binding to the Vasn intronic sequence (5'-AGGTGGAGTTTGTGGTCTGG-3') and a reverse primer binding to the neo cassette (5'-TGGGAAGACAATAGCAGGCATGC-3') were used. For the 3' junction, a forward primer binding to the lacZ ORF (5'-CACATGGCTGAATATCGACGG-3') and a reverse primer binding to the 3' downstream external sequence (5'-ACCTGCCTCACATTTGTTCC-3') were selected. Homologously recombined ES cells were aggregated with $\mathrm{C} 57 \mathrm{Bl} / 6$ recipient morulae to obtain chimeras. After germline transmission had been established, mice carrying a Vasn neo-lacz allele were crossed with CMV-Cre transgenic mice (Schwenk et al., $1995)$ to remove the selection cassette. Mice carrying a $\operatorname{Vasn}^{\text {lacz }}$ allele were bred with wild-type NMRI mice, and resulting offspring were genotyped with a three-primer PCR to discriminate between wild-type and heterozygous embryos using wild-type forward primers binding to the Vasn exon 2 (5'-GGCAACTTCTACAGCTCAGG-3') and the lacZ ORF (5'-CACATGGCTGAATATCGACGG-3') for the wild-type and Vasn ${ }^{\text {lacZ }}$ allele, respectively, and a common reverse primer binding to the Vasn 3' UTR region (5'-AGATGAGACCCAGCCCAGAG-3').

\subsection{Detection of lacZ activity}

For detection of $\beta$-galactosidase activity in whole mounts, embryos were dissected in cold PBS, fixed in 4\% PFA/PBS for $1 \mathrm{~h}$ at $4{ }^{\circ} \mathrm{C}$, rinsed three times with rinse buffer ( $5 \mathrm{mM}$ EGTA, $0.01 \%$ deoxycholate, $0.02 \% \mathrm{NP} 40,2 \mathrm{mM} \mathrm{MgCl}$, in $\mathrm{PBS}$ ) for $15 \mathrm{~min}$ at $\mathrm{RT}$, and immersed in staining buffer $\left(5 \mathrm{mM} \mathrm{K} \mathrm{K}_{3}\left[\mathrm{Fe}(\mathrm{CN})_{6}\right], 5 \mathrm{mM}\right.$ $\mathrm{K}_{4}\left[\mathrm{Fe}(\mathrm{CN})_{6}\right], 1 \mathrm{mg} / \mathrm{ml} \mathrm{X}$-gal, in rinse buffer) until an appropriate staining intensity was obtained. For section analysis, embryos up to E11.5 were stained overnight as whole mounts, embedded in paraffin, and sectioned. Older embryos were embedded in TissueTek $^{\circledR}$ OCT compound (Ted Pella) and frozen on a metal block cooled with dry ice and ethanol. Cryostat sections $(14-20 \mu \mathrm{m})$ were fixed on ice in $1 \%$ PFA/PBS for $10 \mathrm{~min}$, washed twice in cold $2 \mathrm{mM} \mathrm{MgCl}_{2} /$ PBS for $10 \mathrm{~min}$, rinsed twice in cold rinse buffer for $10 \mathrm{~min}$, and incubated overnight in staining buffer at $37^{\circ} \mathrm{C}$. All sections were counterstained with eosin and mounted in Entellan ${ }^{\circledR}$ (Merck).

\section{Acknowledgements}

We thank Karol Macura for ES cell-morula aggregation, Sonja Banko and Mirjam Peetz for expert animal caretaking, and Chris Bunce, Alexandra Farrall, Pedro Rocha, and Lars Wittler for critical comments on the manuscript. A.M.K. thanks Ralf Spörle for sharing his knowledge of mouse development.

\section{References}

Caccia, D., Zanetti Domingues, L., Micciche, F., De Bortoli, M., Carniti, C., Mondellini, P., Bongarzone, I., 2011. Secretome compartment is a valuable source of biomarkers for cancer-relevant pathways. J. Proteome Res. 10, 4196-4207.

Chen, L., Yao, J.H., Zhang, S.H., Wang, L., Song, H.D., Xue, J.L., 2005. Slit-like 2, a novel zebrafish slit homologue that might involve in zebrafish central neural and vascular morphogenesis. Biochem. Biophys. Res. Commun. 336, 364-371.

Choksi, S., Lin, Y., Pobezinskaya, Y., Chen, L., Park, C., Morgan, M., Li, T., Jitkaew, S., Cao, X., Kim, Y.S., Kim, H.S., Levitt, P., Shih, G., Birre, M., Deng, C.X., Liu, Z.G., 2011. A HIF-1 target, ATIA, protects cells from apoptosis by modulating the mitochondrial thioredoxin, TRX2. Mol. Cell. 42, 597-609.

George, S.H., Gertsenstein, M., Vintersten, K., Korets-Smith, E., Murphy, J., Stevens, M.E., Haigh, J.J., Nagy, A., 2007. Developmental and adult phenotyping directly from mutant embryonic stem cells. Proc. Natl. Acad. Sci. USA 104, 4455-4460.

Ikeda, Y., Imai, Y., Kumagai, H., Nosaka, T., Morikawa, Y., Hisaoka, T., Manabe, I., Maemura, K., Nakaoka, T., Imamura, T., Miyazono, K., Komuro, I., Nagai, R., Kitamura, T., 2004. Vasorin, a transforming growth factor beta-binding protein expressed in vascular smooth muscle cells, modulates the arterial response to injury in vivo. Proc. Natl. Acad. Sci. USA 101, 10732-10737.

Malapeira, J., Esselens, C., Bech-Serra, J.J., Canals, F., Arribas, J., 2010. ADAM17 (TACE) regulates TGFbeta signaling through the cleavage of vasorin. Oncogene.

Moon, P.G., Lee, J.E., You, S., Kim, T.K., Cho, J.H., Kim, I.S., Kwon, T.H., Kim, C.D., Park, S.H., Hwang, D., Kim, Y.L., Baek, M.C., 2011. Proteomic analysis of urinary exosomes from patients of early IgA nephropathy and thin basement membrane nephropathy. Proteomics 11, 2459-2475.

Schwenk, F., Baron, U., Rajewsky, K., 1995. A cre-transgenic mouse strain for the ubiquitous deletion of loxP-flanked gene segments including deletion in germ cells. Nucleic Acids Res. 23, 5080-5081.

Ypsilanti, A.R., Zagar, Y., Chedotal, A., 2010. Moving away from the midline: new developments for Slit and Robo. Development 137, 1939-1952. 